\title{
Ein Porträt Friedrichs des Großen in Württemberg
}

\author{
Von Inga QuANDEL
}

Die königlichen Wohnräume in der Beletage des Neuen Corps de Logis in Schloss Ludwigburg hatte König Friedrich I. von Württemberg (1754-1816) zu Beginn des 19. Jahrhunderts durch Nikolaus Friedrich von Thouret (1767-1845) neu gestalten und einrichten lassen. Das Schloss diente dem ersten württembergischen Königspaar als Sommerresidenz. Detaillierte Inventarbeschreibungen liefern ein genaues Bild über die Nutzung und Einrichtung der einzelnen Räumlichkeiten. Zahlreiche dort beschriebene Ausstattungselemente wie Möbel, Gemälde, Uhren, Vasen und vieles mehr sind bis heute im Schloss erhalten geblieben. Um einen möglichst authentischen Raumeindruck zu vermitteln, sollen die erhaltenen Mobilien in Zukunft in ihrem ursprünglichen Kontext präsentiert werden. Im Zuge dieser Neukonzeption werden zahlreiche Objekte konserviert, restauriert und dabei eingehend untersucht. So sind für das Aufwartszimmer König Friedrichs I. in einem Inventarband von 18228 Stück Familiengemälde aus dem preußischen Hause $^{1}$ belegt. Hierzu zählt unter anderem ein Porträt Friedrichs des Großen (1712-1786), das 2016 restauriert wurde (Abb. 1).

Das Kniestück zeigt den jungen Friedrich II. nach rechts gewandt, das Gesicht ist im Dreiviertelprofil dargestellt. Sein Blick ist direkt auf den Betrachter gerichtet. Das grau gepuderte Haar ist im Nacken mit einer schwarzen Schleife zusammengebunden. Die feinen Locken fallen ihm lang über den Rücken herab. Friedrich II. trägt einen blauen, an den Ärmeln und den Taschen reich mit silbernen „Brandenbourgs“ verzierten Samtjustaucorps mit schräg geöffneten Dreiviertelärmeln. Darunter wird ein weißes Hemd mit eng anliegenden, spitzenbesetzten Manschetten sichtbar. Über dem Uniformrock glänzt der eiserne Kürass. Die orange Schärpe mit dem Kreuz des preußischen Schwarzen-Adler-Ordens ist ihm um die Schulter, eine weiße Seidenschärpe mit schmaler Goldeinfassung um die Hüfte gebunden. Der karmesinrote Mantel, der ihm locker über den linken Arm gelegt ist, bauscht sich hinter seiner Figur auf. Die linke Hand hat Friedrich II. locker auf den Kommandostab gelegt, der auf einer Böschung aufsteht. Seine Rechte ruht auf einem eisernen Helm mit Messingbeschlägen und Reiherstutz, der ebenfalls auf dem

${ }^{1}$ Ludwigsburg, Residenzschloss, Inventar 1822, Bd. II, fol. $3^{\text {r }}$. 
grasbewachsenen Felsblock liegt. Der Hintergrund öffnet den Blick auf eine düstere Waldlandschaft vor einem finsteren Himmel.

Bislang galt das Bildnis als eine Kopie des Porträts, das der Maler und Architekt Georg Wenzeslaus von Knobelsdorff (1699-1753) Mitte der 1730er Jahre angefertigt hat und das sich heute im Besitz der Stiftung Preußische Schlösser und Gärten Berlin-Brandenburg befindet (Abb.2). Während der Restaurierungsarbeiten ist jedoch am rechten unteren Bildrand, oberhalb der alten Inventarnummer 1783, eine Inschrift zum Vorschein gekommen: ant. Pesne.

Diese Signatur gab Anlass zur weiteren Untersuchung des Werkes vor allem in Hinblick auf die Entstehungsgeschichte und die Provenienz. Ein besonderes Augenmerk liegt hierbei auf den beiden Hofkünstlern Antoine Pesne (1683-1757) und Georg Wenzeslaus von Knobelsdorff. Die Einordnung des Porträts in die Reihe der Bildnisse, die aus der Kronprinzenzeit Friedrichs II. durch diese beiden Künstler entstanden, geben Hinweise auf die Datierung und die Verbreitung des Werkes ${ }^{2}$. Neben der kunsthistorischen Einordnung interessiert vor allem, wie das Porträt an den württembergischen Hof gelangte, wo es noch knapp ein Jahrhundert nach seiner Entstehung das Aufwartszimmer des Königs zierte. Für die Einordnung des Gemäldes in seinen historischen Entstehungskontext bedarf es zugleich eines genauen Blickes auf die preußisch-württembergischen Beziehungen im 18. Jahrhundert ${ }^{3}$.

\section{Kronprinzenbildnisse Friedrichs des Großen}

Der Maler Antoine Pesne wurde am 23. Mai 1683 in Paris geboren. Im Frühjahr 1710 folgte Pesne dem Ruf Friedrichs I. (1657-1713) und siedelte an den Berliner Hof über. Auch nach dem Tod des Königs und der Thronbesteigung seines Nach-

2 Die Werkkataloge und Monographien zu Pesne und Knobelsdorff liefern einen umfassenden Einblick in ihr CEuvre. Siehe: Rainer Michaelis, Antoine Pesne (1683-1757). Die Werke des preußischen Hofmalers in der Berliner Gemäldegalerie, Berlin 2003; „Zum Maler und zum großen Architekten geboren“. Georg Wenzeslaus von Knobelsdorff (1699-1753). Ausstellung zum 300. Geburtstag, Ausstellungskatalog hg. von der Generaldirektion der Stiftung Preußische Schlösser und Gärten Berlin-Brandenburg, Berlin 1999; Helmut Börsch-Supan, Der Maler Antoine Pesne. Franzose und Preuße, Nürnberg 1986; Ekhart Berckenhagen, Werkkatalog, in: Antoine Pesne, hg. von der Verwaltung der ehemaligen Staatlichen Schlösser und Gärten Berlin in Verbindung mit dem deutschen Verein für Kunstwissenschaft, Berlin 1958, S.93-222. Arnold Hildebrand, Das Bildnis Friedrichs des Großen. Zeitgenössische Darstellungen, Berlin/Leipzig ${ }^{2} 1942$.

3 Siehe hier vor allem: Ernst Boepple, Friedrich des Grossen Verhältnis zu Württemberg, München 1915; Joachim BRÜSER, „C’est le plus abominable et détestable pays du monde“ - Die Erziehung Carl Eugens und seiner Brüder am preußischen Hof 1741 bis 1744, in: Aufgeklärte Herrschaft im Konflikt. Herzog Carl Eugen von Württemberg 1728-1793 (Geschichte Württemberg. Impulse der Forschung 1), hg. von Wolfgang MäHRLE, Stuttgart 2017, S. 21-48. 
folgers Friedrich Wilhelms I. (1688-1740) im Jahr 1713 blieb der Maler trotz erheblicher Gehaltskürzungen als einer von wenigen Hofkünstlern in seinem Amt. Während seiner Zeit als Hofmaler fertigte Pesne zahlreiche Bildnisse der Mitglieder des preußischen Hofes an.

Der zweite genannte Porträtist Friedrichs II. ist der am 17. Februar 1699 geborene Architekt und Maler Hans Georg Wenzeslaus Freiherr von Knobelsdorff. Nachdem Knobelsdorff seinen militärischen Dienst quittierte, widmete er sich seinen künstlerischen Neigungen und freundete sich mit Pesne an. Mit diesem Mentor studierte Knobelsdorff die Lehren der Malerei und übte sich mit unterschiedlichen Materialien an zahlreichen Genres. Tilo Eggeling schreibt über die Beziehung der beiden Künstler zueinander: „Weit prägender war der [...] Einfluß seines Freundes Antoine Pesne (1683-1757), dessen Schüler er seit etwa 1737 war. Es kann daher nicht wundern, daß er sich schon in der Thematik seiner Bilder an seinem Lehrer orientierte. Dies gilt insbesondere für seine Porträts. " ${ }^{4}$ Von Knobelsdorff sind unter anderem Bildnisse Friedrich Wilhelms I. sowie auch seines Sohnes bekannt. Mit der Übernahme der Regierung 1740 übertrug ihm Friedrich II. die Oberaufsicht über die Architektur und Gärten.

Für die zeitliche Einordnung des Ludwigsburger Gemäldes sind vor allem die Porträts interessant, die in den 1730 er Jahren entstanden und in einer Vielzahl überliefert sind. Hierbei lassen sich mindestens drei Bildnistypen charakterisieren, deren Erfindung auf Antoine Pesne zurückzuführen ist.

\section{Das Rehabilitationsporträt ${ }^{5}$}

Als erstes in dieser Reihe ist das sogenannte „Rehabilitationsporträt“ aus dem Jahr $1733 \mathrm{zu}$ nennen. Von diesem sind einige Versionen überliefert, die in der Ausschnittauswahl und zum Teil auch in den Details variieren. In der Eremitage in Bayreuth ist das wohl früheste und qualitativ beste Stück erhalten ${ }^{6}$. Die Bezeich-

${ }^{4}$ Tilo Eggeling, Knobelsdorffs malerischer Geschmack - „gout pittoresque“, in: „Zum Maler und zum großen Architekten geboren“. Georg Wenzeslaus von Knobelsdorff (1699-1753). Ausstellung zum 300. Geburtstag, Ausstellungskatalog hg. von der Generaldirektion der Stiftung Preußische Schlösser und Gärten Berlin-Brandenburg, Berlin 1999, S. $21-52$, hier S. 25.

${ }^{5}$ Die Typisierung der Porträts erfolgt nach: Stella Junger, Präsentation im Bildnis deutscher Fürsten des 18. Jahrhunderts. Sachsen, Polen, Bayern und Brandenburg-Preußen zwischen Absolutismus und Aufklärung (Wissenschaftliche Studien der Westfälischen Wilhelms-Universität Münster, Reihe 6, Bd. 10), Münster 2011, S. 287-303.

${ }^{6}$ Antoine Pesne, Kronprinz Friedrich von Preußen, 1733, Öl/Leinwand, $141 \times 111 \mathrm{~cm}$, Bayreuth Eremitage, Altes Schloss, Vorzimmer der Markgräfin, Bayerische Staatsgemäldesammlungen, Inv.-Nr.7226. Berckenhagen (wie Anm.2) Nr.115 fb. Insgesamt führt Berckenhagen bereits 13 Versionen dieses Typs auf. Siehe auch: Junger (wie Anm. 5) S. 287; Abb. II.3.01. 
nung ,Rehabilitationsporträt‘ leitet sich von den Ereignissen des Frühjahrs $1730 \mathrm{ab}$. Friedrich II. hatte den Plan gefasst, der Erziehungsgewalt seines despotischen Vaters Friedrich Wilhelm I. zu entfliehen. Dabei wurde er von seinem Vertrauten Hans Hermann von Katte (1704-1730), Leutnant in der preußischen Armee, und seinem Pagen unterstützt. Am 5. August unternahm der Kronprinz einen Fluchtversuch aus seinem Reisequartier in Steinsfurt im Kraichgau, der jedoch kläglich misslang. Dem Fluchthelfer Katte wurden Landesverrat und Desertion vorgeworfen, und auf Befehl Friedrich Wilhelms I. verurteilte man ihn zum Tode. Der König zwang seinen Sohn bei der Hinrichtung seines Freundes in der Festung von Küstrin, wo Friedrich II. inhaftiert war, zuzusehen. Vermutlich erhielt Pesne den Auftrag für die Darstellung Friedrichs II. als Feldherr anlässlich seiner Entlassung aus der Gefangenschaft.

Der Kronprinz trägt hier den blauen Uniformenrock des Infanterieregiments Nr. 15. Die zahlreichen Stiche des Porträts belegen, dass es sich um ein offizielles Staatsbildnis des Thronfolgers handelt, das weite Verbreitung finden sollte ${ }^{7}$ Zu diesem Bildnis ist ein Pendant erhalten - das früheste bekannte Porträt Elisabeth Christines von Braunschweig Wolfenbüttel-Bevern (1715-1797), das sie als Kronprinzessin von Preußen zeigt ${ }^{8}$. Damit diente das Bildnis im doppelten Sinn der Staatspropaganda: Zum einen wurde die Versöhnung von Vater und Sohn bekannt gegeben, zum anderen die gerade erfolgte Hochzeit im Bildmedium festgehalten. Die Eintracht und Stärke des preußischen Herrscherhauses wird auf diese Weise demonstriert.

\section{Das erste Rheinsberger Porträt}

Der zweite Porträttypus entstand wohl um 1736 als Friedrichs II. Schwester Wilhelmine von Bayreuth (1709-1758) ihren Bruder um ein aktuelles Bildnis bat ${ }^{9}$. In der Kleidung entspricht das Porträt dem oben vorgestellten Ludwigsburger

7 Vgl. Helmut BöRsch-Supan, Friedrich der Große im zeitgenössischen Bildnis, in: Friedrich der Große in seiner Zeit, hg. von Oswald Hauser (Neue Forschungen zur brandenburg-preussischen Geschichte, Bd. 8), Köln 1987, S.255-270, hier S.261. Die Bildkomposition sowie auch die Haltung des Prinzen verweisen unverkennbar auf das von Jean-Baptiste van Loo geschaffene Staatsporträt des jungen französischen Thronfolgers Ludwigs XV. aus dem Jahr 1728 (Versailles, Schloss, Saal des Überflusses).

8 Antoine Pesne (?), Königin Elisabeth Christine als Braut, 1733, Öl/Leinwand, $83 \times 65$ cm, Potsdam, Neues Palais, GK I 2895. Berckenhagen (wie Anm. 2) Nr. 84 a; Hildebrand (wie Anm.2) S. 98.

9 Antoine Pesne, Kronprinz Friedrich von Preußen, 1736, Öl/Leinwand, $143 \times 113 \mathrm{~cm}$, ehemals Berlin, Schloss, GK I 479, derzeit Niederlande, Haus Doorn. „Ich bitte Dich, mir Dein Bild zu schicken, von Pesne als Kniestück gemalt. Das was ich besitze, gleicht Dir nicht mehr im entferntesten." Zitiert nach Hildebrand (wie Anm. 2) S.105f. Siehe auch: Junger (wie Anm. 5) S.296; Abb. II.3.14. 
Bildnis. Doch hier ist Friedrich in einer Dreiviertelansicht gezeigt. Mit der rechten Hand umschließt er fest den Kommandostab, in der linken hält er den Degenknauf. Der reich mit Federn verzierte Helm steht aufrecht auf dem Felsen zu Friedrichs II. Linken. Am 10. März 1736 schrieb Friedrich II. in einem Brief an seine Schwester: „Pesne bietet seine ganze Kunst auf, um Deinem Befehl gemäß ein gutes Bild von mir zu malen; ich bitte ihn nicht so viel Gewicht auf die Gesichtszüge zu legen, sondern die Gefühle auszudrücken, die ich für Dich hege, damit sie Dir stets gegenwärtig sein mögen. “10 Diese Aussage verdeutlicht, dass es Friedrich II. weniger auf eine exakte Darstellung seiner Physiognomie ankam, als vielmehr auf die Botschaft an den Adressaten. Die Entrückung seiner Person aus der Alltäglichkeit in ein ideales Dasein erfolgt hierbei auf Kosten seiner individuellen Züge. Die Absendung des Porträts an seine Schwester verschob sich jedoch, da Friedrich II. seinen Hofmaler Antoine Pesne mit der Anfertigung zahlreicher gewünschter Kopien beauftragte ${ }^{11}$.

\section{Das letzte Kronprinzenporträt}

Bereits 1738 schuf der Hofmaler einen weiteren Porträttypen, auf dessen Eigenhändigkeit und Datierung ein von Pesne persönlich geschriebener Text auf der Rückseite des Bildes Aufschluss gibt ${ }^{12}$. Die Frontalität und der kleine Bildausschnitt rücken den Kronprinzen nahe an den Betrachter. Das Gesicht, nahezu en face dargestellt, wird von den grau gepuderten Locken umschlossen. Unter der schweren, eisernen Rüstung schaut nur am Hals der Kragen eines weißen Hemdes hervor. Über der linken Schulter liegt der leuchtend rote Hermelinmantel, auf dem das Eiserne Kreuz des Schwarzen-Adler-Ordens prangt. In seiner ungewöhnlich frontalen Wendung zum Betrachter greift Pesne den Darstellungsmodus auf, der bereits 1729 bei einem Staatsporträt Friedrich Wilhelms I. zur Anwendung kam ${ }^{13}$. Somit wird die Kontinuität der preußischen Politik im Bildmedium thematisiert.

10 Friedrich der Große und Wilhelmine von Bayreuth. Jugendbriefe Bd.1, hg. von Gustav Bertold Volz, Leipzig 1924, S. 312.

11 Am 3. April 1736 schreibt Friedrich: „Mein Bild kann ich dir erst nach der Revue schicken; denn Pesne muss eine Unmenge Kopien machen, die nicht früher fertig werden [...] Zitiert nach Berckenhagen (wie Anm.2) S. 130.

12 peint par Antoine Pesne peintre du Roy / anno 1738 zitiert nach Hildebrand (wie Anm.2) S. 107 f. Antoine Pesne, Kronprinz Friedrich von Preußen, 1738, Öl/Leinwand, $79 \times 64 \mathrm{~cm}$, Potsdam, Neues Palais, GK I 10153. Berckenhagen (wie Anm. 2) Nr.115 k. Siehe auch: Junger (wie Anm. 5) Abb. II.3.18.

13 Vgl. Junger (wie Anm. 5) S. 302. 


\section{Das Ludwigsburger Porträt}

Einem weiteren Porträttypus aus dieser Zeit ist auch das Bildnis Friedrichs II. aus dem Besitz der Staatlichen Schlösser und Gärten Baden-Württemberg zuzuordnen. 1975 hatte die Stiftung Preußische Schlösser und Gärten Berlin-Brandenburg ein solches von der Hand des Georg Wenzeslaus von Knobelsdorff erworben. Das Gemälde stammt aus dem Besitz der Nachfahren Dietrich Freiherr von Keyserlingks (1698-1745), einem der engsten Freunde Friedrichs II. Er war bereits 1729 als Gesellschafter des Kronprinzen tätig und spielte vor allem in der Rheinsberger Hofgesellschaft eine bedeutende Rolle. Helmut Börsch-Supan zur Folge beauftragte Friedrich II. im Mai 1737 Keyserlingk ein gerade fertig gestelltes Porträt von Knobelsdorff an ihren gemeinsamen Freund Voltaire zu übermitteln ${ }^{14}$. Das Aussehen des Werkes ist nicht beschrieben, doch hält der Autor diesen Typus aufgrund seiner „französischen Note“ für sehr wahrscheinlich. Er äußert die Vermutung, dass Keyserlingk als Anerkennung für die Ausführung des Auftrags ein gleiches Porträt erhalten haben könnte. Stella Junger bewertet das „von den Vorstellungen des Vaters abweichende[s] bekenntnishafte[s] Porträt“ ${ }^{15}$ ganz ähnlich und geht davon aus, dass dieser Typus vornehmlich für enge Freunde Friedrichs II. geschaffen wurde.

Diese These scheinen auch weitere Versionen des Werkes zu belegen. Im Jahr 1740, zur Zeit seiner Thronbesteigung, schenkte Friedrich II. dem Vater seines Fluchthelfers Hans Heinrich von Katte (1681-1741) eines dieser Bildnisse ${ }^{16}$. Es wird ebenfalls der Hand Georg Wenzeslaus von Knobelsdorffs zugeschrieben. Der Unterschied zu dem Ludwigsburger Werk liegt vor allem in der Kleidung Friedrichs II. Statt des reich verzierten Samtjustaucorps trägt er den blauen Uniformrock mit roten Ärmelaufschlägen des Infanterie-Regiments Nr.15. Catharina Luise

14 Vgl. Helmut Börsch-Supan, Bemerkungen zu einem wiedergefundenen Bildnis Friedrichs des Großen von Georg Wenzeslaus von Knobelsdorff, in: Festschrift für Otto von Simson zum 65. Geburtstag, hg. von Lucius Grisebach/Konrad Renger, Frankfurt a. M./Berlin/Wien 1977, S.398-411, hier S. 398.

15 Junger (wie Anm. 5) S. 295. Börsch-Supan vermutet ebenfalls, dass Friedrich II. dieses Porträt besonders gerne mochte, da es den Uniformvorschriften seines Vaters widersprach. „Die Feldherrenpose hat in diesem Bildnis etwas Spielerisches. Knobelsdorffs Begabung für das bewegte Ornament entfaltet sich vor allem in Mantel, Schärpe und Haar, aber auch in den Händen. Damit sind die künstlerischen Neigungen des Modells eher als seine staatsmännischen Interessen illustriert.“ BÖRSCH-SupaN (wie Anm. 6) S.262.

16 Georg Wenzeslaus von Knobelsdorff, Kronprinz Friedrich von Preußen, um 1737, Öl/ Leinwand, 142 x 119 cm, Familienstiftung der Gans Edlen Herren zu Putlitz. Siehe: Lothar Lambacher, Eltern und Kinder, in: Kriegsgericht von Köpenick! Anno 1730: Kronprinz Katte - Königswort, Ausstellungskatalog hg. von Jürgen Kloosterhuis/Lothar LambaCHER, Berlin 2011, S. 38-69, hier S. 40. Auf der Burg Hohenzollern ist ebenfalls ein Bildnis dieses Typus erhalten, das Friedrich im blauen Uniformenrock zeigt. Es wurde von JungER (wie Anm. 2) S. LXXVIII Antoine Pesne zugeschrieben und um 1736 datiert. 
Gebhardine von Wins ( $†$ 1834) berichtete über diese Schenkung: „Das Bild hat der König meinem Großvater [...] geschickt, gleich nach seiner Thronbesteigung. So viel ich weiß, hat er aber nichts dazu geschrieben, wohl um die alte Wunde nicht aufzureißen."17 Zudem erhob Friedrich II. den Vater seines zehn Jahre zuvor hingerichteten Fluchthelfers in den erblichen Grafenstand. „Sowohl die Schenkung des Gemäldes wie die Standeserhebung darf wohl als ein besonderer Ausdruck der gnädigen Gesinnung des Königs gegenüber der Familie von Katte betrachtet werden." 18

Die Erweiterung zu einem ganzfigurigen Porträt „hat ein schwächerer, unbekannter Künstler von dem im Neuen Palais in Potsdam bewahrten Bild versucht. “19 Nach Arnold Hildebrand stammt das Bildnis von einem Schüler Antoine Pesnes. Ein um 1840 entstandenes Aquarell von Carl Friedrich Wilhelm Klose belegt, dass dieses Porträt zusammen mit weiteren Preußenbildnissen in der Prinzeß-MarieKammer im Berliner Schloss aufhängt war ${ }^{20}$. Diese Räumlichkeiten wurden durch den Hofbaumeister Andreas Schlüter beim Ausbau des Königsschlosses für das Kronprinzenpaar Friedrich Wilhelm I. und seine Gemahlin Sophie Dorothea (1687-1757) angelegt und eingerichtet. In einem dieser Räume kam Friedrich der Große zur Welt. Das ursprünglich rechteckige Werk wurde 1888 in die Wand über dem Kamin der Bibliothek des kaiserlichen Appartements im Berliner Schloss eingelassen ${ }^{21}$.

Zusammenfassend lässt sich also festhalten, dass es sich bei dem Ludwigsburger Porträttypus im Gegensatz zu den anderen dreien weniger um ein offizielles Staatsporträt als vielmehr um ein privates Porträt handelte, das den Wünschen Friedrichs II. entsprach und sich in den Details nicht an die streng reglementierten Darstellungskonventionen des Vaters hielt. Friedrich II. scheint diese Bildnisse hauptsächlich an Personen verschenkt zu haben, zu denen er eine hohe emotionale Bindung hatte.

Die Datierung des Typus wird kontrovers diskutiert ${ }^{22}$. Als Urbild wurde bislang häufig eine ganzfigurige Ölskizze Friedrichs des Großen von Georg Wenzeslaus

17 Zitiert nach Lambacher (wie Anm. 16) S. 40.

18 Ebd., S. 40.

19 Börsch-Supan (wie Anm. 14) S. 403. Er beurteilt dieses Unterfangen als missglückt. Häufig wird das Werk Knobelsdorff zugeschrieben. Schüler des Antoine Pesne, Kronprinz Friedrich von Preußen, 1736, Berlin, Schloss, $127 \times 104 \mathrm{~cm}$, GK I 1015. BerCKENHAgen (wie Anm. 2) Nr.115 g. Siehe: Hildebrand (wie Anm. 2) Taf. 23.

${ }^{20}$ Das Aquarell befindet sich heute in der Sammlung Georg Schäfer in Schweinfurt. Siehe: Der frühe Realismus in Deutschland 1800-1850. Gemälde und Zeichnungen aus der Sammlung Georg Schäfer, Schweinfurt, Ausstellungskatalog hg. vom Germanischen Nationalmuseum Nürnberg, Nürnberg 1967, Nr. 140.

21 Vgl. Hildebrand (wie Anm. 2) S. 105.

22 Vgl. Friedrich Benninghoven, Erziehung zum Regenten, in: Friedrich der Große. Ausstellung des Geheimen Staatsarchivs Preußischer Kulturbesitz anläßlich des 200. Todestages König Friedrichs II. von Preußen, Ausstellungskatalog hg. von Friedrich Benning 
von Knobelsdorff angesprochen (Abb.2) 23 $^{23}$ Sie befand sich mit Sicherheit im 18. Jahrhundert in der Wohnung der Königin Elisabeth Christine, Gemahlin Friedrichs II., im Berliner Schloss. Charles Foerster schlug aufgrund des Aussehens Friedrichs II. eine Entstehungszeit „um 1738“ vor ${ }^{24}$. Hildebrand hingegen plädiert wegen der Darstellung der Haartracht, „die noch ohne ausgeprägten Scheitel ist“ ${ }^{\text {25, }}$ für eine frühere Datierung um 1736. Börsch-Supan geht davon aus, dass die Skizze an den ersten hier besprochenen Bildnistypus „Bayreuth“ von Pesne anknüpft und daher vermutlich um 1734 entstanden ist. Stella Junger setzt die Entstehung des Prototyps etwas später um das Jahr $1735 \mathrm{an}^{26}$. Auch wenn eine genaue Datierung nicht durch schriftliche Quellen belegt werden kann, scheint die Entstehung in der Mitte der 1730 er Jahre eindeutig.

Nun stellt sich allerdings die Frage nach dem Urbild. Da sich Knobelsdorff in den ersten Jahren seiner Künstlerkarriere stark an Pesne orientierte, wäre ebenfalls möglich, dass die ursprüngliche Idee vom Meister und nicht von seinem Schüler ausgegangen ist. So erinnert die Haltung Friedrichs II. stark an die Rötelzeichnung eines unbekannten Feldherrn, die Pesne bereits um 1715 fertigte ${ }^{27}$. Die Kopfgestaltung hingegen entspricht der des Ludwigsburger-Porträts. Der Mohr sowie auch die Gestaltung des Hintergrundes sind auf ein Bildnis von Leopold Fürst zu Anhalt-Dessau zurück zu führen, das als ein Hauptwerk Pesnes von 1710 gilt $^{28}$. Knobelsdorff ersetzt das Zelt auf der linken Seite durch einen Waldrand. Damit handelt es sich bei dem als Skizze angesprochenen Werk von Knobelsdorff um eine Art Pasticcio, bei dem der Künstler auf die Ideen seines Lehrers zurückgreift und daraus ein eigenständiges Werk kreiert. Sollte das Ludwigsburger-Bildnis das

Hofen/Helmut Börsch-Supan/Iselin Gundermann, Berlin 1986, S. 51, Nr. 86. „Das Bildnis zeigt Friedrich so, wie ihn Knobelsdorff etwa beim Feldzug am Rhein oder zu Anfang der Rheinsberger Zeit gesehen hat. [...] Entstanden ist es nach der Teilnahme am Feldzug am Oberrhein." Ebenfalls um 1735: Junger (wie Anm. 5) S. LXXVII.

${ }^{23}$ Börsch-Supan hält das kleinformatige Werk für ein vollwertiges Bild. Dafür sprechen seiner Meinung nach die sorgfältige Durchgestaltung des Kopfes sowie auch der Umstand, dass das Bild 1786 von Friedrich Nicolai im Esszimmer der Königin Elisabeth Christine erwähnt wird. BöRsch-SupAN (wie Anm. 14) S. 403.

${ }^{24}$ Charles F. Foerster, Antoine Pesne 1683-1757. Ausstellung zum 250. Geburtstag, Ausstellungskatalog hg. von der Verwaltung der Staatlichen Schlösser und Gärten in historischen Wohnräumen des Berliner Schlosses, Berlin 1933, Nr. 124.

${ }^{25}$ Hildebrand (wie Anm. 2) S. 103.

26 „Die Datierung des Prototyps ist überzeugend um 1735 festgelegt. Zum einen verweist darauf die Haartracht, Friedrich trägt hier noch keinen ausgeprägten Mittelscheitel, wie er Ende der Dreißigerjahre aufkommt. Zum anderen deutet die Form der ,Brandenbourgs', die bereits Rokokocharakter angenommen haben, auf die Mitte der Dreißigerjahre." JungeR (wie Anm. 5) S. 290.

27 Antoine Pesne, unbekannter Feldherr, um 1715, Rötelzeichnung, 30,4 ×21,8 cm, Wien, Albertina, Inv.-Nr.12003. Siehe: Junger (wie Anm. 5) Abb. II.3.09.

${ }^{28}$ Das Porträt ist verschollen und nur in einem Schabkunstblatt von 1798 überliefert. Johann Joseph Freidhoff, Leopold, Fürst von Anhalt-Dessau bei der Belagerung von Aire (1710), 72,5 × $50 \mathrm{~cm}$. Berckenhagen (wie Anm. 2) Nr. 202. 
einzige durch Pesne signierte Werk dieses Typus sein, könnte es sich hierbei also um die Urfassung handeln.

Wahrscheinlicher ist jedoch, dass das Urbild am Berliner Hof bewahrt wurde. Ein ganz ähnliches Porträt war bis 1945 im Besitz der Stiftung Preußische Schlösser und Gärten Berlin-Brandenburg, ist jedoch seitdem verschollen (Abb.3). Das Gemälde befand sich 1942 im Treppenflur des Alten Palais in Berlin ${ }^{29}$. Ursprünglich war es vermutlich im Berliner Schloss zusehen. Frauke Mankartz bemerkte, dass die Porträts Friedrichs des Großen von Antoine Pesne aus der Kronprinzenzeit auch noch nach seiner Thronbesteigung weiterverwendet wurden ${ }^{30}$. Die Gesichtszüge wurden dann dem aktuellen Alter angepasst - dies scheint auch bei dem Ludwigsburger Porträt erfolgt zu sein.

\section{Die Beziehungen zwischen Württemberg und Preußen}

Wie und wann das Bildnis an den württembergischen Hof gelangte, ist bislang nicht eindeutig zu belegen. Der Porträttypus wurde zwar bereits um 1735 entwickelt, doch hat Friedrich II. Hans Heinrich von Katte, dem Vater seines Fluchthelfers, noch Jahre später ein solches Bildnis zukommen lassen. Die Verbreitung des Porträts fällt somit in den Zeitraum eines Regierungswechsels am württembergischen Hof.

Bereits unter dem württembergischen Herzog Eberhard Ludwig (1676-1733) wurde 1716 ein Freundschaftsvertrag mit Friedrich Wilhelm I. geschlossen ${ }^{31}$. In diesem Jahr erfolgte ebenfalls die Vermählung der Hohenzollernprinzessin Henriette Marie von Brandenburg-Schwedt (1702-1782), Cousine des preußischen Königs, mit dem Erbprinzen Friedrich Ludwig von Württemberg (1698-1731). So entstanden erste Bande zwischen den beiden Dynastien. Antoine Pesne fertigte ein Bildnis des frisch vermählten Erbprinzenpaares, wahrscheinlich im Auftrag von Friedrich Wilhelm I. $\mathrm{an}^{32}$. Als nach vierzehn Jahren Ehe immer noch kein

29 Vgl. Hildebrand (wie Anm. 2) S. 105.

30 Vgl. Frauke Mankartz, Die Marke Friedrich. Der preußische König im zeitgenössischen Bild, in: Friederisiko. Friedrich der Große die Ausstellung, Ausstellungskatalog hg. von der Generaldirektion der Stiftung Preußische Schlösser und Gärten Berlin-Brandenburg, München 2012, S. 204-221, hier S.205. So diente auch das hier als letztes Kronprinzenporträt angesprochene Bildnis noch 1742 als Vorlage für ein Geschenk an den französischen Hof. Siehe: Junger (wie Anm. 5) S. 301.

31 Vgl. Carmen Winkel, Im Dienste seiner Majestät: Netzwerke im Offizierskorps als Mittel der Außenpolitik (Militär und Gesellschaft in der Frühen Neuzeit, Heft 14.1), hg. von Gundula Gahlen/Carmen Winkel, Potsdam 2010, S. 59-84, hier S. 76.

32 Antoine Pesne, Henriette Marie von Brandenburg-Schwedt und Friedrich Ludwig von Württemberg, um 1716, Öl/Leinwand, $237 \times 183 \mathrm{~cm}$, Staatliches Museum Schwerin, Inv.-Nr. G 230. Siehe: Harald Schukraft, Kleine Geschichte des Hauses Württemberg, Tübingen 2006, S. 114. 
Thronfolger aus dieser Verbindung hervorgegangen war, reiste der Soldatenkönig im August 1730 persönlich an den württembergischen Hof. Er befürchtete, dass die Regierung an den zum katholischen Glauben konvertierten Carl Alexander von Württemberg-Winnental (1684-1737) fallen könnte. Dieser Besuch wird vor allem Friedrich dem Großen im Gedächtnis geblieben sein, der seinen Vater auf der Reise in den Süden begleitete. Am 5. August unternahm der Kronprinz nämlich in Steinsfurt bei Sinsheim den verhängnisvollen Fluchtversuch.

Bei einem Gegenbesuch Eberhard Ludwigs 1731 in Berlin wurde das Freundschaftsbündnis erneuert und um den Passus erweitert, dass sich die beiden Parteien im Falle eines Krieges gegenseitige Unterstützung zusicherten und sich zusätzlich dem Schutz der protestantischen Kirche verschrieben. Bei diesem Aufenthalt hatte Antoine Pesne ein Porträt Eberhard Ludwigs angefertigt, das bis heute im Residenzschloss Ludwigsburg erhalten ist ${ }^{33}$. Das Feldherrenbildnis zeigt den Herzog in einer roten, goldbestickten Galauniform. Den rechten Arm hat er mit dem Marschallstab seitlich ausgestreckt, die linke Hand umfasst den Degengriff. Eberhard Ludwig ist hier in seinem höchsten Rang als kaiserlicher General mit dem Stern des preußischen Schwarzen-Adler-Ordens und dem juwelenverzierten Kreuz des württembergischen St. Hubertus Jagdordens dargestellt. Heute erinnert das Bildnis, fest in die Wandvertäfelung seines Schlafzimmers im Neuen Corps de Logis in Ludwigsburg eingelassen, an den großen Bauherrn ${ }^{34}$.

Die Befürchtungen Friedrich Wilhelms I. sollten sich zwei Jahre später bewahrheiten, als der Katholik Carl Alexander den württembergischen Thron bestieg. Er regierte das Land bis zu seinem unerwartet gekommenen Tod am 12. März 1737. Sein Sohn Carl Eugen übernahm im Alter von neun Jahren die Regierung. Bis zu seiner Volljährigkeit wurde das Land jedoch unter der Administration der Herzöge Carl Rudolf von Württemberg-Neuenstadt (1667-1742) und Carl Friedrich von Württemberg-Oels (1690-1761) geführt.

33 Antoine Pesne, Eberhard Ludwig von Württemberg, 1731, Öl/Leinwand, $154 \times$ $125 \mathrm{~cm}$, Ludwigsburg, Residenzschloss, KRGT 5772. Berckenhagen (wie Anm. 2) Nr.79a. Siehe: Paul SAUER, Musen, Machtspiel und Mätressen. Eberhard Ludwig - württembergischer Herzog und Grüner Ludwigsburgs, Tübingen 2008, S. 9.

${ }^{34}$ Im Hauptstaatsarchiv in Stuttgart sind die Berechnungen der Auslagen des Barons von Röder, Erboberstallmeister von Herzog Eberhard Ludwig, aus den Jahren 1731 - 33 für Ankäufe von Kunstgegenständen erhalten. Laut Rechnung vom königl. Hofmabler Antoni Pesne, vor unterschiedliche gelieferter Mablereyen dd: Berlin den 26. Sept. 1733. 81. Rthl. (HStAS A 210 III Bü 130). Die handschriftliche Rechnung an den Geheimrat Weinreier für une copie de Portray de son altesse serenisime Madame La Duchesse de Wirttenberg jusqua jenaux von Pesne selber ist ebenfalls überliefert. Die Duchesse de Wirttenberg war zu diesem Zeitpunkt noch die Gemahlin Eberhard Ludwigs, Johanna Elisabeth von BadenDurlach. Bei dem Original, das Pesne laut Rechnung ausbesserte, könnte es sich um das Gegenstück zu dem in Ludwigsburg erhaltenen Feldherrenbildnis des Herzogs handeln (KRGT 5772). In der Rechnung steht des Weiteren geschrieben: plus pour avoir fait racomoder Loriginal du portray de Son altesse madame La Duchesse (HStAS A 210 III Bü 130). 
Eine sehr intensive Verbindung der beiden Höfe ist vor allem mit dem Regierungsantritt des Preußenkönigs Friedrichs II. nachzuweisen. Dieser fasste bereits 1740 den Entschluss, die katholischen Prinzen aus Württemberg - Carl Eugen und seine beiden jüngeren Brüder - zu sich zu nehmen. Trotz der offensichtlichen konfessionspolitischen Hintergedanken stimmte die Mutter der Prinzen, Maria Augusta von Thurn und Taxis (1706-1756), einem Aufenthalt ihrer Kinder in Berlin $\mathrm{zu}^{35}$. Die Herzogin musste sich in dieser Zeit vor allem gegen die territorialen Eliten behaupten, die gegen die Politik ihres verstorbenen Ehemannes opponierten. Zunächst zielten ihre Machtbestrebungen auf die Unterstützung des Kaiserhofes in Wien, was ihr jedoch nur geringe Vorteile einbrachte, sodass sie 1737 einen radikalen Richtungswechsel vollzog und sich nach Preußen hin orientierte ${ }^{36}$. Diese Entscheidung ist wohl vor allem mit dem hohen Rang des preußischen Königshauses wie auch mit der Hoffnung auf eine Verbesserung ihrer ungünstigen Mitvormünderin-Stellung in Württemberg zu begründen.

Auf ein Schreiben von Herzogin und Herzog-Administrator an Friedrich II. vom 22. November 1741 erwiderte dieser, es hätte ihm nie eine größere Freude bereitet werden können, „und man habe ihm keinen größeren Beweis aufrichtigsten Vertrauens geben können, als das man ihm die Prinzen zur Erziehung überlasse“ 37 . So kam es, dass Carl Eugen im Alter von knapp 14 Jahren zusammen mit seinen Brüdern für etwas mehr als zwei Jahre zur Erziehung an den Hof Friedrichs

35 Siehe vor allem: Ulrike SEEgER, Von Preußen lernen? Die Hofkunst Herzog Carl Eugens von Württemberg zwischen Berlin und Paris, in: Wie friederizianisch war das Friederizianische? Zeremoniell, Raumdisposition und Möblierung ausgewählter europäischer Schlösser am Ende des Ancien Régime. Beiträge einer internationalen Konferenz vom 2. Juni 2012, Friedrich300-Colloquien 6, hg. von Henritte Graf/Nadja GeIssLER: http:// www.perspectivia.net/publikationen/friedrich300-colloquien/friedrich_friderizianisch/ seeger_hofkunst (Abruf: 12.10.2017), bes. S.1-5.

${ }^{36}$ Vgl. Gabriele Haug-Moritz, Maria Augusta, in: Das Haus Württemberg, hg. von Sönke Lorenz/Dieter Mertens/Volker Press, Stuttgart/Berlin/Köln 1997, S. 256-258.

37 Nach Boeppel (wie Anm.3) S.14. Der Kontakt zwischen Friedrich II. und Maria Augusta von Thurn und Taxis scheint in dieser Zeit besonders intensiv gewesen zu sein. Im Hauptstaatsarchiv in Stuttgart ist ein Brief Friedrichs II. an die Herzogin vom 30. März 1742 erhalten, in dem der König versichert, sich für die Belange der Herzogin stark zu machen und sie sowie auch ihre Angehörigen immer zu beschützen. Enfin à l'egard $d u$ dernier article comme le Roi, se fait une etude particuliere, de marquer en toute rencontre à Madame la Duchesse combien ses interêts lui sont chers, Sa Majesté s'engage de la proteger de toutes ses forces, toutes les fois, qu'il en sera besoin, contre quiconque tentera de l'attaquer, de l'inquieter, où de lui nuire, soit en haine des demarches, que Son Altesse Serenissime a faites pour complaire aux intentions $d u$ Roi, où sur d'autres pretextes où raisons quelconques. Sa Majesté prend pareillement sous sa protection Rö̈ale, toutes les personnes que Son Altesse Serenissime a employeés jusqu'ici à l'execution de ses vuës ou qu'elle y employera, où agréera a son service dans la suite; et Elle ne souffrira point, que par cette raison, elles soyent rechercheés ou inquieteés de quelque façon que se soit. Sa Majesté faisant donner au surplus a Madame la Duchesse les assurances les plus fortes de son affection et amitié constante, et de son estime parfaite et distinguée. Fait à Olmutz, ce 30 mars, 1742 (HStAS G 197 Bü 18). 
des Großen geschickt wurde. Hier bot er den Prinzen ein komfortables Leben im Palais Schwerin mit zahlreichen Gelegenheiten an Opern, Komödien, Konzerten und anderen Vergnügungen teilzunehmen. Um die enge Verbindung zu stärken, griff Friedrich II. auf ein bewährtes Mittel der Außenpolitik zurück und verlieh den Prinzen sowie auch ihrer Mutter preußische Regimenter. „Der Erbprinz Karl Eugen erhielt das Infanterieregiment Nr.46, sein Bruder Ludwig Eugen das Dragoner Regiment Nr. 2 [...]. Die Herzoginwitwe Maria Augusta wurde ehrenhalber zum Oberst des Dragonerregiments Nr. 12 ernannt, das man aus den württembergischen Diensten übernommen hatte. Als erste Frau erhielt sie zudem den Schwarzen-Adler-Orden. “38 Als die verwitwete Herzogin Maria Augusta im Februar nach Berlin reiste, wurden ihr zu Ehren eine Reihe von Festlichkeiten veranstaltet.

Trotz aller Bemühungen Friedrichs II., die jungen Prinzen an den preußischen Hof zu binden, baten sie aufgrund von Heimweh bereits im Frühjahr 1743 um die Erlaubnis zur Rückkehr nach Württemberg. Daraufhin erwirkte Friedrich II. beim Wittelsbacher Kaiser Karl VII. die vorzeitige Mündigsprechung - im Alter von 16 Jahren trat Carl Eugen 1744 die Regierung in Württemberg an. Ehe er Berlin verließ, bekam er den sogenannten „Fürstenspiegel oder Unterweisung des Königs für den jungen Herzog Karl Eugen von Württemberg“ überreicht. Hierin erteilte Friedrich II. seinem Zögling Anweisungen und Ratschläge für die Regierung seines Landes. Er beginnt mit folgenden Worten: „Mit dem Anteil, den ich an Ihrer Mündigkeitserklärung hatte, verbindet sich mein Interesse an einem glücklichen Verlauf Ihrer Regierung. Ich stelle mir geradezu vor, das Gute oder Schlimme, das daraus entspringt, werde sich irgendwie auch auf mich zurückwenden. In diesem Sinne halte ich mich für verpflichtet, Ihnen mit freundschaftlichem Freimut meine Anschauungen über den neuen Stand, in den Sie nun eintreten, anzusprechen. “39

Zudem beschlossen Friedrich II. und die Herzogin von Württemberg, Carl Eugen mit der Nichte des Königs, Prinzessin Friederike Elisabeth Sophie von Brandenburg-Bayreuth (1732-1780), zu verheiraten. Die Verlobung fand auf der Rückreise des Herzogs nach Württemberg am 21. Februar 1744 statt. Erst vier Jahre später wurden die beiden am Hof in Bayreuth getraut. Friedrich der Große erschien nicht zu der Feierlichkeit, sondern ließ sich von seinen Brüdern vertreten. Das Verhältnis zwischen den beiden verschlechterte sich nach der Abreise Carl Eugens aus Berlin zusehends. So schrieb der Preußenkönig an seine Schwester: „Ich mache mir keine Hoffnung, daß er meiner Nichte die Treue halten wird. Sein Charakter ist mir zur Genüge bekannt. “40

Noch in Berlin fertigte Antoine Pesne 1744 ein Porträt des jungen Herzogs, das er mit in seine Heimat nahm und das bis heute in seinem Appartement in Schloss

38 Winkel (wie Anm. 31) S. 77.

39 Der Fürstenspiegel ist online einsehbar unter http://gutenberg.spiegel.de/buch/ -5328/1 (Abruf: 12.10.2017).

40 Zitiert nach: Karlheinz WAgNer, Herzog Karl Eugen von Württemberg. Modernisierer zwischen Absolutismus und Aufklärung, München 2001, S. 18. 
[Die Abbildungen 1-4 können aus rechtlichen Gründen online nicht bereitgestellt werden.]

Abb. 1: Antoine Pesne, Kronprinz Friedrich von Preußen, um 1736, Öl/Leinwand, $140 \times 104 \mathrm{~cm}$

(Aufnahme: Silke Schick; Ludwigsburg, Residenzschloss, KRGT 5808).

Abb. 2: Georg Wenzeslaus von Knobelsdorff, Friedrich II. der Große, um 1735 (?), Öl/Leinwand, 58,5 × 48,5 cm

(Aufnahme: Daniel Lindner; Potsdam, Schloss Sanssouci,

Stiftung Preußische Schlösser und Gärten Berlin/Brandenburg GK I 1203).

Abb. 3: Antoine Pesne, Kronprinz Friedrich von Preußen

(Vorlage: Stiftung Preußische Schlösser und Gärten Berlin/Brandenburg

GK I 172, Verbleib unbekannt).

Abb. 4: Antoine Pesne, Herzog Carl Eugen von Württemberg, 1744, Öl/Leinwand, $288 \times 200 \mathrm{~cm}$ (Vorlage: Staatliche Schlösser und Gärten Baden-Württemberg; Ludwigsburg, Residenzschloss, KRGT 5805). 
Ludwigsburg erhalten geblieben ist (Abb.4) ${ }^{41}$. Das Ganzfigurenbildnis zeigt den sechzehnjährigen Herzog leicht nach rechts gewendet. Das Gesicht wird von einer weißen Lockenperücke gerahmt, sein Blick fixiert den Betrachter. Er trägt einen samtblauen Uniformenrock, der an den Säumen reichlich mit Gold bestickt ist. Unter den umgeschlagenen Ärmeln schauen die eng anliegenden, mit Spitze besetzten Manschetten hervor. Um die Schulter ist das rote Band mit dem Stern vom württembergischen Jagdorden gelegt, an die Brust der Orden vom Goldenen Vlies geheftet. Den rechten Arm hat Carl Eugen selbstbewusst in die Seite gestützt, die linke Hand liegt - ähnlich wie auch auf dem Porträt Friedrichs II. - auf einem Helm mit weißem Federbusch auf. Dieser steht auf einem prunkvollen RokokoKonsoltisch, auf dem weitere Herrscherinsignien aufgestellt sind: Ein mit Hermelin verbrämter Samtmantel, der Herzogshut, ein Dreispitz und weiße Handschuhe. Nach der vorzeitigen Mündigsprechung durch den Kaiser ist dieses Porträt das erste, das den jungen Carl Eugen in der Rolle des Landesregenten zeigt ${ }^{42}$.

In den folgenden Jahren musste Friedrich II. zusehen, wie sein Zögling trotz seiner Lehren und Ermahnungen einen Lebensstil pflegte, der das Land Württemberg durch ungeheuren finanziellen Aufwand an den Rand des Ruins führen sollte $e^{43}$.

\section{Historische Einordnung}

Im Nachlassinventar Carl Alexanders, das erst sechs Jahre nach seinem Tod erstellt wurde, ist unter den Gemälden und Porträts ein Stück aufgeführt, dessen knappe Beschreibung auf das Bildnis passt: 1. langlecht Stk: 4Eckigt 1. Portrait von

41 Die Datierung ist durch eine Inschrift a Berlin en 1744 gesichert.

${ }^{42}$ Ein weiterer Hinweis auf die Verbindung der beiden Dynastien und auf ein Originalgemälde Pesnes am württembergischen Hof lässt sich in einer Liste des Ludwigsburger Gemälde-Galerie Sturzes von 1751 finden, die sich ebenfalls im Hauptstaatsarchiv in Stuttgart erhalten hat. Während die Verzeichnisse fast ausschließlich aus reinen Nummernverweisen bestehen, ist neben der 1777 vermerkt: Markgraf Christian von Bayreuth von Anton Pesne gemablt ein vorzüglich schönes Portrait von ganz vortreflichen Effect. So das es unter den besten Bildern gezählt werden kann (HStAS A 21 Bü 528, fol.25r). Wie auch das Bildnis der Duchesse de Wirttenberg, ist das hier genannte Porträt nicht im Bestand der Staatlichen Schlösser und Gärten Baden-Württemberg erhalten.

${ }^{43}$ Das Zerwürfnis zwischen den beiden führte sogar soweit, dass sich Carl Eugen im Siebenjährigen Krieg mit den Feinden Friedrichs II. verbündete und mit 12.000 Mann gegen ihn ins Feld rückte. „Damit erntete er freilich weder Ehre noch Ruhm, und nach der Schlacht bei Torgau beauftragte Friedrich, nicht ohne Ironie, den in preußische Diensten stehenden Prinzen Friedrich Eugen von Württemberg, die fliehenden Truppen Herzog Karls nicht sowohl zu verfolgen als vielmehr, heim zu geleiten'“ A. Von Winterfeld, Friedrich der Große und Herzog Karl Eugen von Württemberg, in: Deutsche Revue 23,2 (1898) S. 186-191, hier S. 189. 
dem jetzt regierend König in Preußen als ein Kniestück ${ }^{44}$. Da das Inventar in das Jahr 1743 datiert, muss Friedrich der Große gemeint sein. Die explizite Herausstellung jetzt lässt vermuten, dass das Porträt nicht den regierenden König, sondern den Kronprinzen zeigt, also vor 1740 entstanden ist. Gehen wir davon aus, dass damit das Kronprinzenbildnis Friedrichs II. im Residenzschloss in Ludwigsburg gemeint ist - vor allem, da aus dem Bestand der Staatlichen Schlösser und Gärten Baden-Württemberg kein weiteres Porträt des Königs aus dieser Zeit bekannt ist - muss das Werk spätestens im Jahr 1743 an den württembergischen Hof gekommen sein. Da das Porträt in dem Nachlassinventar Carl Alexanders erwähnt wird, muss es entweder an ihn selber gegangen sein, oder wahrscheinlicher nach seinem Tod an seine Frau Maria Augusta von Thurn und Taxis (1706-1756). Wie bereits erwähnt, hatte Friedrich II. großes Interesse daran, die drei Söhne der beiden an den preußischen Hof in seine Obhut zu holen. Das Bildnis könnte als eine Art Werbung gedient haben, die Maria Augusta dazu bewegen sollte, ihre Kinder in die Hände des starken und selbstbewussten Königs zu geben. „Im Spannungsfeld zwischen Courtoisie, politischer Strategie und Reputation gelegen, bildeten großzügige Geschenke auch für den auf Sparsamkeit bedachten Friedrich den Großen ein unverzichtbares und wirksames Mittel der Diplomatie. "45 Damit wurde das Bildnis als eine nonverbale, symbolische Kommunikationsform genutzt, das zum einen dem Rang des Adressaten schmeichelte und gleichzeitig zur Steigerung der eigenen Reputation eingesetzt wurde.

Sofern das Bildnis tatsächlich als Geschenk an Maria Augusta von Thurn und Taxis gegangen ist, würde dies die Autorisierung des Werkes durch Pesnes Signatur erklären. So schreibt Wilhelmine von Bayreuth, die ältere Schwester Friedrichs Großen, in ihren Memoiren, dass Pesne ein Bildnis der Königin fertigte. Zeitgleich bat der Generalfeldmarschall Friedrich Wilhelm Grumbkow die Königin um ein Porträt, „welches, wie er sagte, seinem Hause den größten Glanz verleihen würde. " 46 Doch statt ihm das Original zukommen zu lassen, schickte sie ihm eine Kopie dessen, „weil sie nur Fürstinnen Originale gäbe. “47 Von allen bekannten Adressaten, an die ein Bildnis des Ludwigsburger Typus geschenkt wurde, war der württembergische Hof der Ranghöchste. Den Worten Königin Sophie Dorotheas,

${ }^{44}$ HStAS G 196 Bü 30, fol. 479r.

45 Jeanette Opalla, Das Geschenkwesen am friderizianischen Hof. Absicht und Botschaft, in: Friedrich der Große und der Hof. Beiträge einer internationalen Konferenz vom 10./11. Oktober 2008, Friedrich300-Colloquien 2, hg. von Michael KaIsER/Jürgen LuH, S.1: http://www.perspectivia.net/publikationen/friedrich300-colloquien/friedrichhof/Opalla_Geschenkewesen (Abruf: 17.10.2017).

46 Wilhelmine von Bayreuth. Eine preußische Königstocher. Glanz und Elend am Hofe des Soldatenkönigs in den Memoiren der Markgräfin Wilhelmine von Bayreuth, hg. von Ingeborg Weber-Kellermann, aus dem Französischen von Annette Kolb, Frankfurt am Main ${ }^{11}$ 2016, S. 129.

47 Weber-Kellermann (wie Anm. 46) S.129. 
Mutter von Friedrich II. und Wilhelmine, folgend, hätte den Württembergern also der Erhalt eines Pense'schen Originals zugestanden.

\section{Fazit}

Das Bildnis Friedrichs des Großen in Ludwigsburg stellt sowohl unter kunsthistorischen als auch unter historischen Aspekten eine bemerkenswerte dynastiegeschichtliche Quelle dar. Im Kontext der zahlreichen Kronprinzenporträts der 1730 er Jahre ist es ein weiteres Zeugnis für die Bildnispropaganda des preußischen Königshofes. Sowohl Antoine Pesne als auch Georg Wenzeslaus von Knobelsdorff sorgten mit der Produktion und Reproduktion immer neuer Bildnistypen für die Entstehung einer nonverbalen Kommunikationsform, die politische Statements vermitteln konnte. Der Vergleich der Kronprinzenbildnisse hat gezeigt, dass der Fokus je nach Habitus und Darstellungstradition auf unterschiedliche Bildaussagen gerichtet werden konnte. Während das sogenannte „Rehabilitationsporträt“ die Versöhnung mit dem Vater und die Heirat publik machen sollte, verdeutlicht das letzte Kronprinzenporträt nachdrücklich die Kontinuität der preußischen Regierung. Dabei diente das Zurücktreten individueller Züge der Idealisierung und Erhebung des Herrschers.

Mit einer solchen Ehrung stellte Friedrich II. die Bedeutung des kleinen württembergischen Hofes deutlich hervor. Vielleicht hat er sein Bildnis genutzt, um die Herzogin Maria Augusta davon zu überzeugen, ihre Kinder zur Erziehung an den preußischen Hof zu geben. Die kurze Notiz im Nachlassinventar Carl Alexanders von 1743 stützt diesen Verdacht. Auch wenn sich das Verhältnis zwischen den beiden Dynastien nach Carl Eugens Abreise aus Berlin 1744 rapide verschlechterte, war das Bildnis Friedrichs des Großen noch über seinen Tod hinaus zu Beginn des 19. Jahrhunderts im Aufwartszimmer des ersten württembergischen Königs in seiner Sommerresidenz in Ludwigsburg zu sehen. Hier verwies es, neben zahlreichen weiteren Porträts aus dem preußischen Hause, auf die bedeutenden Ahnen Friedrichs I. von Württemberg. Dieser sollte dann am 1. Januar 1806 die Königswürde annehmen und die Bildnisse geschickt zur Legitimation seiner Macht einsetzen. Denn sowohl seine Mutter als auch seine Tante waren preußische Prinzessinnen und Nichten des großen Königs gewesen. 